\title{
QUEEN'S
UNIVERSITY
BELFAST
}

\section{Distinguishing between similar tubular objects using pulse reflectometry: a study of trumpet and cornet leadpipes}

Buick, J. M., Kemp, J., Sharp, D. B., Van Walstijn, M., Campbell, D. M., \& Smith, R. A. (2002). Distinguishing between similar tubular objects using pulse reflectometry: a study of trumpet and cornet leadpipes.

Measurement Science and Technology, 13(5), 750-757.

\section{Published in:}

Measurement Science and Technology

Queen's University Belfast - Research Portal:

Link to publication record in Queen's University Belfast Research Portal

\section{General rights}

Copyright for the publications made accessible via the Queen's University Belfast Research Portal is retained by the author(s) and / or other copyright owners and it is a condition of accessing these publications that users recognise and abide by the legal requirements associated with these rights.

Take down policy

The Research Portal is Queen's institutional repository that provides access to Queen's research output. Every effort has been made to ensure that content in the Research Portal does not infringe any person's rights, or applicable UK laws. If you discover content in the Research Portal that you believe breaches copyright or violates any law, please contact openaccess@qub.ac.uk. 


\title{
Distinguishing between similar tubular objects using pulse reflectometry: a study of trumpet and cornet leadpipes
}

\author{
J M Buick ${ }^{1}$, J Kemp ${ }^{1}$, D B Sharp ${ }^{2}$, M van Walstijn ${ }^{1}$, \\ D M Campbell ${ }^{1}$ and R A Smith ${ }^{3}$ \\ ${ }^{1}$ Department of Physics and Astronomy, JCMB, The University of Edinburgh, \\ Mayfield Road, Edinburgh EH9 3JZ, UK \\ ${ }^{2}$ Department of Environmental and Mechanical Engineering, Open University, Walton Hall, \\ Milton Keynes MK7 6AA, UK \\ ${ }^{3}$ Richard Smith Musical Instruments, 110 The Vale, Southgate, London N14 6AY, UK
}

Received 13 August 2001, in final form and accepted for publication

11 March 2002

Published 18 April 2002

Online at stacks.iop.org/MST/13/750

\begin{abstract}
This paper considers the measurement of the internal radius of a number of similar, short, tubular leadpipes using pulse reflectometry. Pulse reflectometry is an acoustical technique for measuring the internal bore of a tubular object by analysing the reflections which occur when an acoustical pulse is directed into the object. The leadpipes are designed to form the initial, or lead, part of a trumpet or cornet and their internal radii differ by less than $0.1 \mathrm{~mm}$ between similar pipes. The ability of the reflectometer to detect these small differences, which are considered by players to produce a noticeable difference in the sound of an instrument, are investigated. It is seen that the pulse reflectometer is able to distinguish between leadpipes with different nominal radii varying by as little as $0.03 \mathrm{~mm}$, demonstrating its potential in the study of musical instruments and showing that it can be used as a diagnostic tool by the instrument manufacturer to detect defects which are significant enough to acoustically alter performance. The absolute accuracy of the radius measurements is also considered at the end of the leadpipe, where the uncertainty is $\pm 0.05 \mathrm{~mm}$.
\end{abstract}

Keywords: acoustic pulse reflectometry, bore profile, leadpipes

\section{Introduction}

In the production and assessment of musical wind instruments it is very useful for the manufacturer to be able to investigate the internal bore, since the ultimate musical performance of the instrument is determined to a great extent by this profile. Although the relationship between the bore shape and the acoustics of the instrument is very complicated, the ability to obtain a detailed description of the internal profile of the instrument can be useful to the manufacturer, particularly as a diagnostic tool enabling comparisons to be made between 'good' and 'bad' instruments. Comparisons of this type can pinpoint regions where action can be taken by the manufacturer to improve the performance of a 'bad' instrument. In this paper we consider the study of a number of leadpipes for both trumpets and cornets. These correspond to the initial portion of the trumpet or cornet and have been designed by the manufacturer with a series of slightly different bores in order to change the musical qualities of the instrument.

For many years the internal bore of musical instruments has been measured using tools such as calipers. While these can give an accurate measure of the bore, it is generally not possible to access the whole of the instrument and so an incomplete profile is obtained. To produce a detailed profile in the accessible regions requires a large number of single measurements and can be time consuming. The range of accessibility can be extended by inserting objects of a known size (or a size which can be measured using calipers) 
into the instrument. This can also be time consuming, and the measurement region is limited to the straight section at either end of the instrument, or adjacent to regions which can be separated (for example the slide on a trombone). Added to this, bends in a tube usually produce an irregular bore section so the only means of measurement remaining is to cut the tube in slices and make many measurements of each segment. An alternative method of obtaining bore profiles is by first measuring the input impedance of the instrument. This is defined as the ratio of the acoustic pressure to the alternating volume velocity at the entrance to the instrument. The bore profile and the input impedance are closely related and, in principle, the bore profile can be derived from an input impedance measurement. To date, attempts at measuring the bore in this manner have proved difficult. Recently [1] it has been shown that bore reconstruction from input impedance is feasible using an optimization technique. This approach is currently being developed further by the author of [1]. Currently its resolution along the axis of the measured instrument is limited by the bandwidth of the system; the accuracy is promising, but the technique is demanding in terms of computational resources. In this paper we consider a third technique: acoustic pulse reflectometry.

Pulse reflectometry is an non-intrusive measuring technique which can be applied to obtain internal measurements of tubular objects. The technique was first developed for seismic studies [2] and has also been applied to airway measurement in medical applications [3-5]. Smith [6] was the first to use impulse reflectometry to detect debris and imperfections in trumpets not visible or measurable by traditional methods. One imperfection of great interest to brass players is valve misalignment and in tests $0.25 \mathrm{~mm}$ was readily detectable. Most recently the technique has been applied to the study of other musical instruments [7-10] as well as general tubular objects $[7,11,12]$. Pulse reflectometry involves inserting a short sound pulse, containing a wide range of frequencies, into the object being measured. The internal reflections of this pulse are then recorded and the input impulse response found. The input impulse response can then be used to calculate the internal bore $[7,11,13,14]$ of the object.

The musical qualities of an instrument (intonation, timbre and response) are strongly dependent on the bore profile of the internal air column. The smaller brass musical instruments, such as a trumpet or cornet, can be characterized by three main sections of brass tube. The most visible section of a trumpet is the bell flare, which is attached to a cylindrical section (with a diameter of approximately $11.8 \mathrm{~mm}$ ) incorporating valves (allowing the musician to play a chromatic scale) and a slide for fine tuning to a standard frequency. This section is matched to the end of the mouthpiece (approximately $7 \mathrm{~mm}$ diameter) with a $250 \mathrm{~mm}$ tapered tube called the leadpipe (pronounced 'leedpipe') or mouthpipe. Smith and Daniell [15] studied the effect on musical qualities of small changes of the order of $0.1 \mathrm{~mm}$ to the bore shapes of trumpets. Due to a proportional effect, these changes have a far greater effect on the smallerdiameter sections such as the leadpipe than on any other part of the instrument, and it is clear from tests that certain players can detect these small differences. Consequently the manufacturer Smith-Watkins Brass [16] provides clients with a choice of 15 different leadpipe profiles that are interchangeable on their model of trumpet. These tapered tubes are made by 'lead drawing' i.e. forcing an annealed brass tube onto a specially shaped hardened steel mandrel by passing both through a lead block. Since there is full control of the manufacture and final gauging of each pipe, Smith-Watkins Brass is confident of repeating the same item first made 16 years ago. In fact in 1999, Smith was awarded the status of Millennium Product for the interchangeable leadpipe system employed on his 'Soloist' cornet [16]. A cornet has a longer (approximately $360 \mathrm{~mm}$ ) leadpipe than a trumpet; since the cornet leadpipe is bent into a large semicircle, internal gauging is impossible by traditional means such as calipers.

A number of the Smith-Watkins Brass leadpipes were used in this study. The trumpet leadpipes are identified by number and come in three families: $\{10,12,14,16\}$, $\{32,34,36,38\}$ and $\{64,66,68,70,72\}$, produced on mandrels I, II and III respectively. Within each family the radius of the pipe increases with the pipe number. Each pipe has the same length and large end radius. The cornet leadpipes are based on five shapes which are labelled, in increasing radius, R0, A0, G0, T0 and K0. Pipes G2 and G4 are also constructed with the same shape as G0 but with slightly larger radii. Similar pipes K2, K4, A2, R2 and T4 are also produced. Note that the numbers are used to label the pipes and have no precise meaning other than the general trend that, within a family, a larger number corresponds to a larger pipe.

Since any manufacturer is primarily interested in observing the difference between either 'similar' pipes (which are intended to be identical) or 'different' pipes (designed to produce a different sound), we concentrate on determining if the pulse reflectometer is capable of these measurements. Any measuring device must be capable of detecting such differences if it is going to be useful for comparing musical instruments and aiding manufacturers. The accuracy of the measured profile is also considered at the end of the pipes, where it can be checked using calipers.

\section{Pulse reflectometry}

The aim of pulse reflectometry is to obtain the internal radius of a tubular object. This is done by determining the object's input impulse response, which is then analysed to determine the internal bore profile.

\subsection{The input impulse response}

The input impulse response is the reflection sequence which would be obtained if an acoustical impulse were fired into the object. For example, consider an object consisting of three cylindrical sections $c_{1}, c_{2}$ and $c_{3}$, shown in figure 1 . When an acoustical impulse propagates through such a tube it will be partially reflected and partially transmitted at each of the radius changes (including the open end); the partial reflections and transmissions at the step $c_{1}: c_{2}$ between cylinders $c_{1}$ and $c_{2}$ are shown in figure 1 . Thus the input impulse response will be a series of impulse-like peaks corresponding to reflections at the steps $c_{1}: c_{2}, c_{2}: c_{3}, \ldots$ and also multiple reflections occurring, for example, by the input impulse being transmitted at $c_{1}: c_{2}$, reflected at $c_{2}: c_{3}$ and finally transmitted at $c_{1}: c_{2}$ towards the source. The shape of each of the peaks constituting the input 


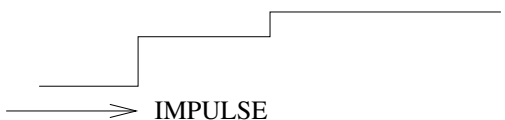

PARTIAL REFLECTION $\longleftarrow \longrightarrow$ PARTIAL TRANSMISSION

$\mathrm{c}_{1}$

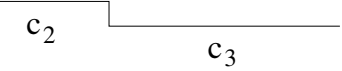

Figure 1. An object consisting of three cylinders $c_{1}, c_{2}$ and $c_{3}$. An impulse signal entering the object is shown, as are the partial reflection and partial transmission at $c_{1}: c_{2}$.

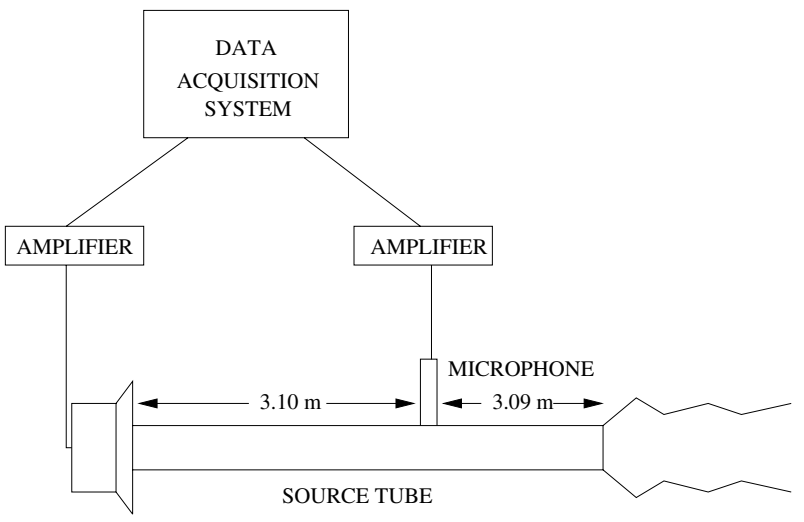

SPEAKER

OBJECT

Figure 2. A schematic diagram of the pulse reflectometer.

impulse response will be slightly different from the impulse due to the losses experienced by the pulse as it propagates in the object.

It is not possible to measure the input impulse response directly since it is impossible to create an ideal acoustic impulse. A schematic diagram of the apparatus used to find the input impulse response is shown in figure 2. The initial pulse is produced by sending a square pulse of duration $80 \mu \mathrm{s}$ to the speaker. The resulting acoustical pulse from the driver propagates along the source tube, past the microphone and into the object. Reflections from the object propagate back along the source tube and are detected by the microphone which is embedded in the wall of the source tube so that the front face of the microphone matches the wall as closely as possible. The signal from the microphone is then amplified and sampled at $50 \mathrm{kHz}$. Here 1024 samples of the reflected signal are recorded giving a time-series of the reflections lasting approximately $20 \mathrm{~ms}$. The length of the source tube and the position of the microphone are selected to ensure that only reflections of the object are recorded and not the input pulse or secondary reflections from the speaker. The apparatus is shown in figure 3.

Using the technique described above it is possible to measure the object reflections; however, this is not the input impulse response because the initial acoustical pulse is not an impulse function. To calculate the input impulse response function we need to deconvolve the measured reflection with the input pulse. Thus the input impulse response is given by the inverse Fourier transform of $I I R(\omega)$ where

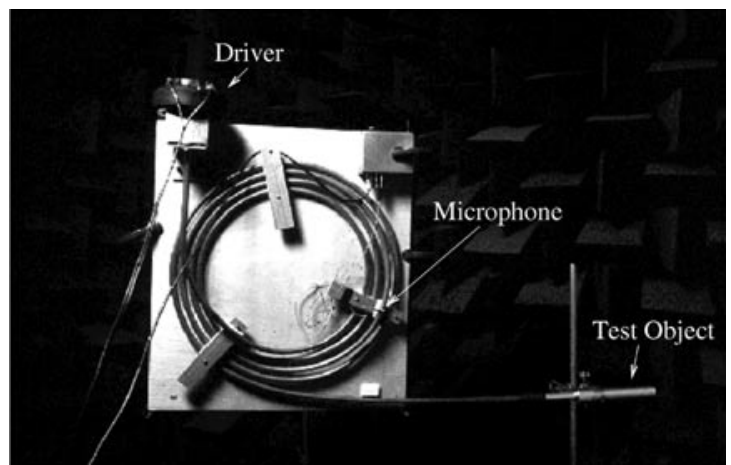

Figure 3. The pulse reflectometer.

$$
I I R(\omega)=\left[\frac{R(\omega) I^{*}(\omega)}{I(\omega) I^{*}(\omega)+q}\right],
$$

$R(\omega)$ is the Fourier transform of the measured reflections, $I(\omega)$ is the Fourier transform of the input pulse and $q$ is a small constraining factor to prevent division by zero. The input pulse was measured by removing the object and replacing it with a rigid cap which terminates the source tube and produces a perfect reflection of the input pulse. This ensures that any signal losses in the tube, between the microphone and the object/rigid termination, are accounted for in the deconvolution. The signal-to-noise ratio was improved by taking 1000 averages of both signals before performing the deconvolution and the measurements were performed in an anechoic chamber.

Finally, it is important to ensure that there is no DC component in the recorded signals; this derives not from any acoustical effects but from the electronics between the microphone and the computer. The DC value imposes an incorrect increase or decrease on the radius (since it is not present in the acoustical signal) which is added consecutively to each point along the object in the final reconstruction. Thus even a very small DC value can add a large error to the radius measurement at the far end of the tube. To find the value of the added DC shift a tube of length $403 \mathrm{~mm}$ and constant radius is inserted between the source tube and the object. The 'DC tube' now becomes part of the object being measured in that it corresponds to the first $403 \mathrm{~mm}$ of the reconstruction. With the 'DC tube' in place the input impulse response is calculated as before using equation (1). Now, it is know that no reflections will occur during the first $2 l / c$ seconds, where $l$ is the length of the 'DC tube' and $c$ is the speed of sound. The average value of the input impulse response is found during this time and corresponds to the DC value. The DC value is then subtracted from the whole signal. This has been shown to be an effective method for removing the DC term $[7,11]$.

\section{Bore reconstruction}

The discrete input impulse response is a time-series of reflections from the object. These reflections occur whenever there is a change in impedance due to the tube increasing or decreasing in radius. The objects being considered generally have a smoothly varying radius. In order to evaluate the internal bore we approximate the object by a series of small cylinders $c_{1}, c_{2}, \ldots$, each of length $c \delta t / 2$, where $\delta t$ is the 


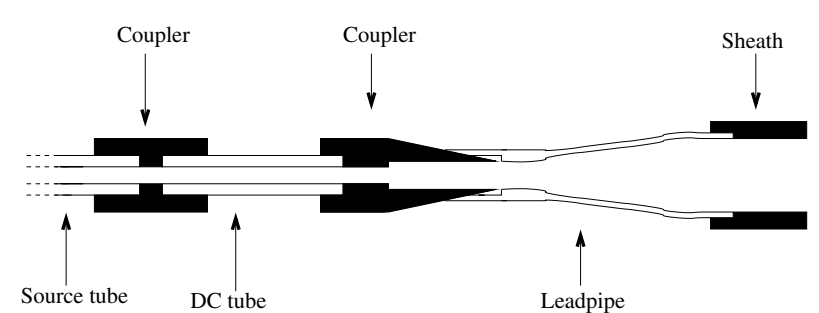

Figure 4. A schematic diagram (not to scale) showing how the source tube, the DC tube, the leadpipe and the sheath are coupled.

sample time. The cross-sectional area of each step can be found provided the initial area of the object is known (or can be measured). Note that the notation used here is similar to the notation introduced in figure 1 ; however, here each cylinder has the same length, $l=c \delta t / 2$ and the radius of each cylinder is the average radius of the actual object over the corresponding length $l$. If an object has a constant radius over several of these interrogation cylinders then there will be no reflections. However each of the 'steps' between the cylinders is still considered separately in the reconstruction algorithm. The first term in the input impulse response series corresponds to the reflection of the impulse at the boundary between the first and second cylinders. Thus the change in impedance, and hence the change in radius, at $c_{1}: c_{2}$ can be found. This can be repeated noting that the subsequent terms in the series are made up from primary reflections and multiple reflections. The sample rate used throughout was $50 \mathrm{kHz}$. Increasing the sample rate to $100 \mathrm{kHz}$ increases the resolution of points along the axis of the object. Other than this, no detectable increase in accuracy was observed due to a decrease in the length of the interrogation cylinders. There is also no increase in the bandwidth since there is no distinguishable signal above $25 \mathrm{kHz}$.

To obtain good accuracy the viscous losses incurred by the sound inside the object must be taken into account. These can be significant for the radius of objects we are considering and are described by Keefe [17] as a function of both the radius and the frequency. Thus in any interrogation cylinder different frequency components within the pulse will be damped at different rates. The resulting change in the shape and amplitude of the pulse is accounted for by a lossy filter. Full details of the lossy filter algorithm can be found in $[13,14]$.

The DC tube was connected to the leadpipe and to the source tube using specially designed couplers, as shown in figure 4. The coupler joining the source tube and the DC tube is designed to keep the radius approximately constant. There is a small step inside the coupler joining the DC tube to the leadpipe. Different couplers were built so that the second internal radius of the coupler approximately matches the internal radius of the leadpipe being measured. Thus the step may be a step up or a step down depending on the leadpipe.

It is well known that a series of ripples occurs in a reconstruction whenever there is a large step in the object's radius, including the open end of a pipe $[7,11]$. This is the Gibbs phenomenon, which arises because of the finite bandwidth of the sampled reflections. To improve the reconstruction near the open end of the leadpipe, a cylindrical sheath was fitted over the open end. This is similar to the continuation of the trumpet which would normally occur at the end of the leadpipe and has the effect of removing the ripples from the reconstruction at the end of the leadpipe. This sheath is also shown in figure 4 .

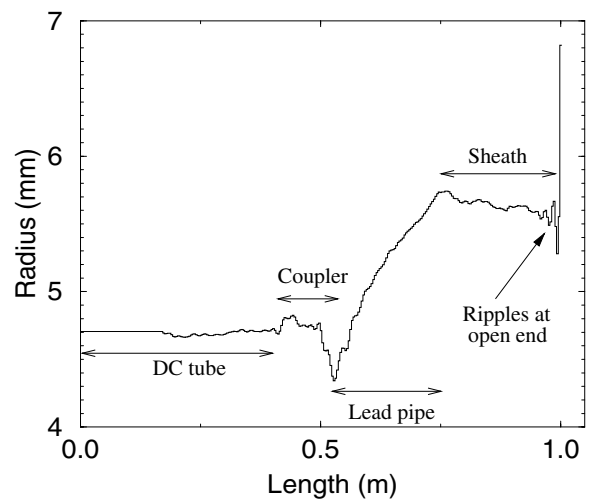

Figure 5. A typical reconstruction of pipe 10. The position of the DC tube, the coupler, the leadpipe and the sheath are marked on the graph. Also marked are the ripples which occur around a large step or an open end, in this case at the open end of the sheath.

\section{Results}

A typical reconstruction of pipe 10 is shown in figure 5 , in which the different portions of the reconstruction are identified. The initial section is the DC tube which should have a fixed radius. The first portion of the DC tube (approximately $16.5 \mathrm{~cm}$, corresponding to $1 \mathrm{~ms}$ of the sampled signal) is fixed at the known radius of the DC tube by the reconstruction algorithm. This removes any anomalous numerical peaks in the initial portion of the deconvolved signal. The second portion of the DC tube reconstruction is calculated from the sampled reflections. Here we see a small variation in the measured radius of considerably less than $0.1 \mathrm{~mm}$. The second section of the reconstruction is the coupler used to connect the DC tube to the leadpipe. It is designed to slip over the DC tube so that the change in the internal radius is minimal. In figure 5 the change is seen to be about $0.1 \mathrm{~mm}$. The small internal step within the coupler can be clearly seen. Inside the initial portion of the leadpipe there is a slot for the trumpet mouthpiece to be inserted. There is a decrease in the radius of the pipe at the end of this slot. On the reflectometer the coupler is inserted into the pipe in the same manner as a mouthpiece; there is therefore an overlap between the coupler and the start of the leadpipe and so the initial portion of the pipe is not measured. The reconstruction of the pipe starts just before the step down in radius at the end of the mouthpiece slot. The variation in the radius of the leadpipe from this step to the end is about $1.5 \mathrm{~mm}$, increasing in a smooth manner along the length of the pipe. Finally we see the sheath at the end of the leadpipe. Ripple is observed on the reconstruction at the open end of the sheath, but not at the end of the leadpipe.

\subsection{Repeatability and measurement accuracy}

When comparing the reconstruction of a number of pipes, either to observe the difference between the pipes, or to compare a number of pipes which are intended to be the same, it is essential to know the accuracy of the measurements so that we can determine which differences in the reconstruction correspond to real differences in the pipes and which to uncertainties in the measuring technique. One type of uncertainty which is present in any reconstruction is a 


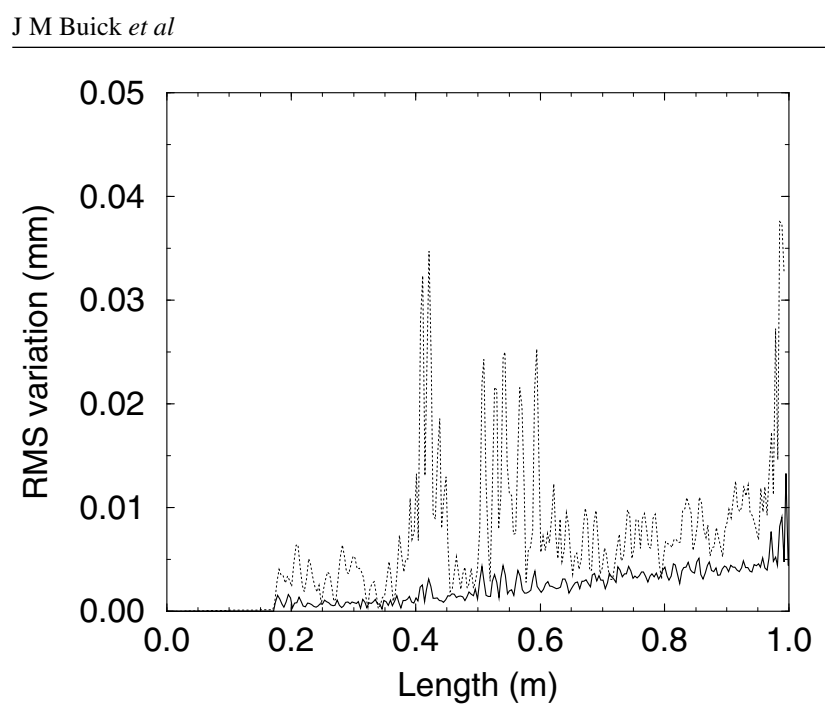

Figure 6. The RMS variation between repeated reconstructions of pipe 10. The reconstructions were repeated using method 1 (thicker, solid curve) and method 2 (thinner, dotted curve).

systematic uncertainty which occurs in every measurement. While it is desirable to be able to measure the actual radius of an object with a high degree of accuracy it should be noted that differences between pipes (which is our primary interest here) can still be observed despite any systematic uncertainties. It is therefore more important here to consider the repeatability and reliability of the measurements; the absolute accuracy of the radius measurements will be considered briefly in section 4.5.

The repeatability of the measurements was studied in two ways. Method 1 involved making ten repeated measurements of the object reflections without removing the pipe or touching the apparatus in any way. Method 2 involved taking ten separate measurements at different times and on different days, this test reflects more closely the conditions under which the reflectometer is likely to be used in a musical instrument workshop.

Methods 1 and 2 were used to examine the repeatability of the technique using pipes 10,38 and G0. The root mean square (RMS) of these variations was calculated and is shown in figures 6 and 7 for pipes 10 and G0 respectively. The results for method 1 , corresponding to ten measurements, are represented by the thicker, solid curve and the results for method 2, corresponding to six of the ten measurements which were selected because they were made at similar temperatures, are represented by the thinner, dotted curve. From figure 5 we can see that there is a step close to 0.4 and $0.5 \mathrm{~m}$ along the object (within the coupler) and that the trumpet leadpipe lies between about 0.5 and $0.75 \mathrm{~m}$. The cornet leadpipe lies between about 0.5 and $0.9 \mathrm{~m}$. There are a number of general features in figures 6 and 7 which were also observed for pipe 38. Firstly, there are a number of peaks at approximately 0.4 and $0.5 \mathrm{~m}$ corresponding to the steps in the coupler and at about $1.0 \mathrm{~m}$ in figure 6 and $0.9 \mathrm{~m}$ in figure 7 corresponding to the open end of the sheath and the end of the cornet leadpipe respectively. The peaks observed for method 2 are due to differences in temperature between the measurements, and differences between the length of the coupler which was inserted into the pipe (seen to vary by up to $2 \mathrm{~mm}$ ). Since the speed of sound is a function of temperature the number of sample points and their separation will be different for reconstructions measured

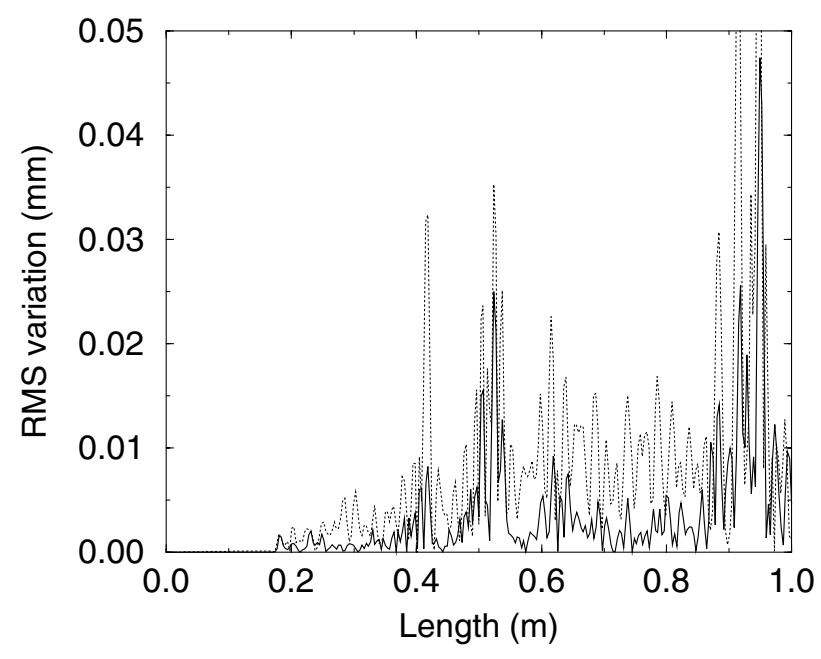

Figure 7. The RMS variation between repeated reconstructions of pipe G0. The reconstructions were repeated using method 1 (thicker, solid curve) and method 2 (thinner, dotted curve).

at different temperatures ${ }^{4}$. In figure 7 there are peaks at 0.4 and $0.5 \mathrm{~m}$ for method 1 suggesting that there may have been a small change in temperature during the half hour taken to obtain the measurements. Except for the peaks the value of the RMS variation is typically about $0.01 \mathrm{~mm}$ in the region of the pipes and generally no greater than $0.02 \mathrm{~mm}$. This suggests that the pulse reflectometer should be able to distinguish between pipes provided they differ by more than about $0.03 \mathrm{~mm}$. It would be expected that variations around regions of step changes in radius would have similar levels of repeatability to other regions under ideal temperature controlled conditions.

\subsection{Trumpet leadpipe reconstructions}

Trumpet leadpipe reconstructions are shown in figure 8, for the two mandrels I and II. Three replicas of pipe 10 (pipes 10a, $10 \mathrm{~b}$ and 10c) and four replicas of pipe 38 (pipes 38a, 38b, 38c and $38 \mathrm{~d}$ ) were reconstructed and are also shown in figure 8 . The replica pipes are intended to be the same; however, they cannot be totally identical. Only the leadpipe is shown in the figures, and the length axis has been re-calibrated so that zero corresponds to the start of the pipe. In some of the pipes where the initial step is larger there is some ripple observed in the reconstruction (see for example pipe 10). This ripple can obscure the measurements slightly, especially close to the step, and may prevent small differences being noticed. In the middle section, between any initial ripple and the convergence at the end of the pipes, the differences between the different sized pipes can clearly be seen within each family. Also the difference between the replica pipes is much smaller than the difference between different pipes (pipes with a different number). Thus, if an unknown pipe was obtained and measured it should be possible to determine which pipe it is.

4 Note that this problem occurs here because of the manner in which the results are compared. Normally the speed of sound is accounted for in the algorithm by varying the spatial separation of the data points. 

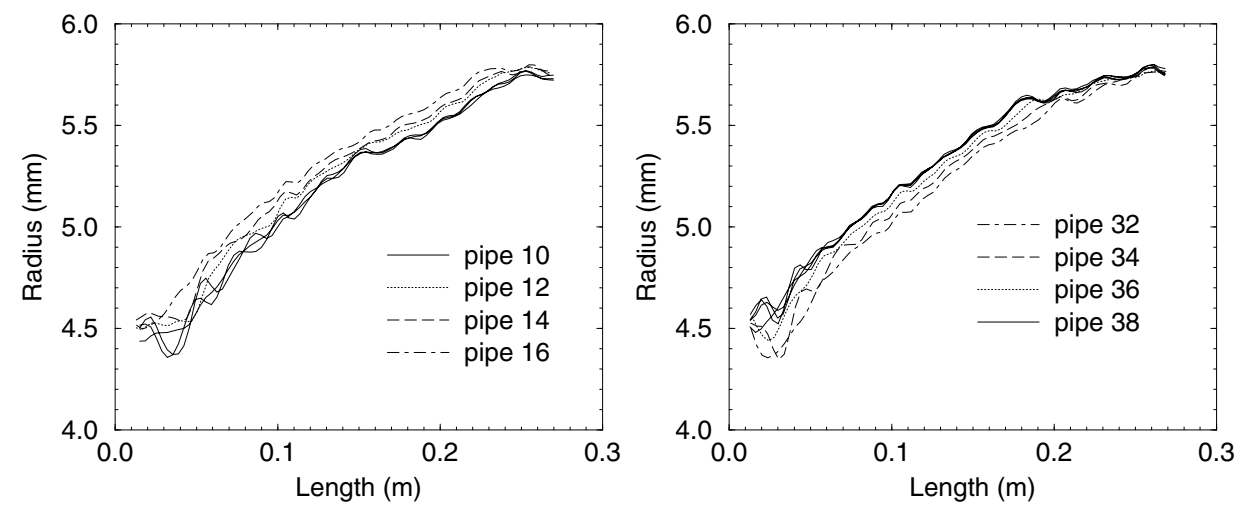

Figure 8. Reconstructions of the four pipes in the mandrel I and II families. Rconstructions of three replicas of pipe 10 and four replicas of pipe 38 are shown.
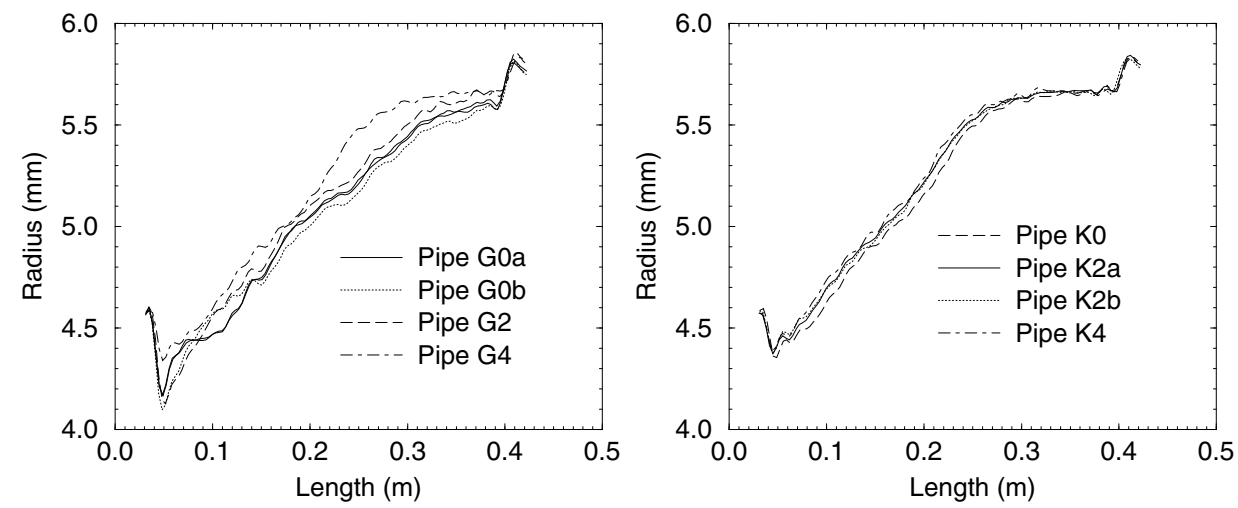

Figure 9. Reconstructions of the $\mathrm{G}$ and $\mathrm{K}$ families of cornet leadpipes. Reconstructions of two replica $\mathrm{G} 0$ and two replica K2 pipes are shown.

\subsection{Cornet leadpipe reconstructions}

We now turn our attention to the cornet leadpipe reconstructions. Figure 9 shows reconstructions of the $\mathrm{G}$ and $\mathrm{K}$ pipes. Both these families contain three pipes labelled 0 , 2 and 4 as their radius increases. We also studied replica G0 and $\mathrm{K} 2$ pipes. It is clear from the figure that the mean radius of the $\mathrm{G}$ family is smaller than that of the $\mathrm{K}$ family, and that the range of radii within the $\mathrm{K}$ family is less than the range within the $\mathrm{G}$ family. Figure 9 shows only a small difference between pipe $\mathrm{K} 0$ and $\mathrm{K} 4$, typically about $0.1 \mathrm{~mm}$. Over the whole length of the pipes the reconstructions for both the K2 pipes are seen to lie between the two curves for $\mathrm{K} 0$ and $\mathrm{K} 4$ and the agreement between the two $\mathrm{K} 2$ pipes is much greater than the agreement between any of the other curves. This suggests a high degree of accuracy in the manufacture of the two K2 pipes with little variation introduced due to the curved shape of the pipes. Figure 9 also shows the pipes increasing in radius from G0 to G4. This is evident everywhere except close to the initial step where the $\mathrm{G} 2$ pipe appears smaller than both the G0 pipes. Since this is close to the step and the differences are small it is hard to determine whether this is due to a small ripple on the reconstruction. There is also a significant difference between the two G0 pipes. In many places the difference between the two G0 pipes is comparable with the difference between the larger $\mathrm{G} 0$ pipe and the $\mathrm{G} 2$ pipe, and in some places it is greater. It is also evident that G0b is smaller than G0a over most of the length, but near the mouthpiece it is greater. Note that two repeated measurements of pipe G0a are shown in figure 9 and they are in good agreement. Repeated measurements of G0b showed similar good agreement with the reconstruction which has been plotted. The reason for the differences between pipe $\mathrm{G0a}$ and G0b being larger than the differences between other replica pipes is not clear. The manufacturer expects the cornet pipes to show a larger deviation due to their curved shape; this could account for the deviation. It is also possible that one of the pipes has been damaged in some way, either during the manufacture, in transit, or during its measurement.

We now look at the full range of the cornet pipes through the five pipes $\mathrm{R} 0, \mathrm{~A} 0, \mathrm{G} 0, \mathrm{~K} 0$ and $\mathrm{T} 0$ shown in figure 10 . The manufacturer [16] indicates that the pipes should increase in radius from the smallest, $\mathrm{R} 0$, through $\mathrm{A} 0, \mathrm{G} 0$ and $\mathrm{K} 0$, to the largest T0. Figure 10 shows both the G0 pipes to demonstrate how they fit into the different families. Clearly the pipes do increase in radius as indicated by the manufacturer and the pipes are distinguishable with the exception of the smallest pipe, R0, which contains a large amount of ripple. The step down at the start of this pipe is larger than any of the others. The amplitude of the ripple is, at its largest, about $0.3 \mathrm{~mm}$ which would be acceptable in many applications; however, here it makes it impossible to compare pipe R0 with the others in a sensible manner, at least for the first half of the pipe. Both the $\mathrm{G} 0$ pipes lie between the $\mathrm{A} 0$ and the $\mathrm{T} 0$ pipes over most of their length, except for a portion between 0.08 and $0.15 \mathrm{~m}$ where G0a becomes significantly smaller than A0. This suggests that G0b may be the 'correct' pipe and G0a the 'damaged' 


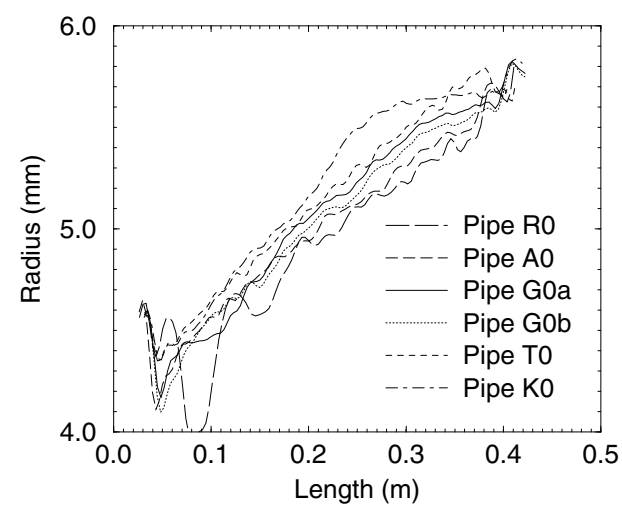

Figure 10. Reconstructions of the cornet leadpipes R0, A0, G0, T0 and K0. The two replica G0 pipes are shown.

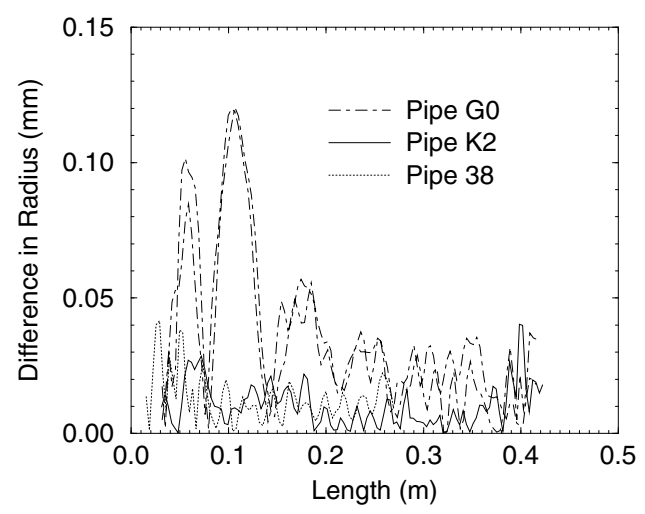

Figure 11. The difference between reconstructions of replicas of pipes $\mathrm{G} 0, \mathrm{~K} 2$ and 38 .

pipe, although the evidence is not conclusive; measurement of another replica G0 pipe would be required to confirm this.

\subsection{Differences between replica pipes}

The replica pipes we had available were 10a, 10b, 10c; 38a, 38b, 38c, 38d; G0a, G0b and K2a, K2b. Repeated measurements of each of these pipes were made and the differences between the reconstructions, for example $10 \mathrm{a}-10 \mathrm{~b}$, were considered. Figure 11 shows one such difference for pipes 38 and $\mathrm{K} 2$ and two repeated differences for pipe G0. The results shown for pipes 38 and K2 are typical of the other results obtained for these pipes and for the results obtained for pipe 10 . The variation between the replica pipes is generally no greater than the variation observed when repeated measurements of a single pipe were considered. Thus any deviation between these replica pipes is too small for the reflectometer to detect. The two repeated comparisons of pipes G0a and G0b show a distinct difference between the pipes which is distinguishable from the variations obtained by repeated measurements of a single pipe. We can therefore say with certainty that there is an observable difference between the two G0 pipes, even although the difference is never greater than $0.12 \mathrm{~mm}$.

\subsection{Accuracy of the measurements}

In the previous sections we have been concerned with comparing the shape of a series of pipes rather than obtaining exact

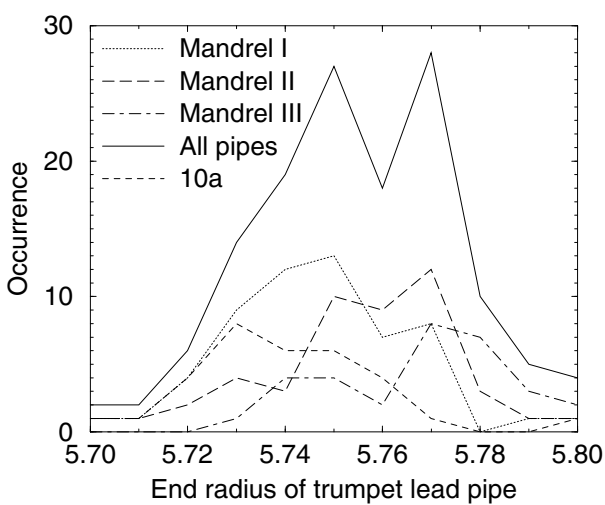

Figure 12. The occurrence of each end radius (rounded to the nearest $0.01 \mathrm{~mm}$ ) for each of the trumpet leadpipe reconstructions. The results are shown in five groups corresponding to all the trumpet pipes, all the pipes constructed from each of mandrel I, mandrel II and mandrel III, and all the reconstructions of the single pipe 10a.

measurements of their radius. To investigate the accuracy of radial measurements made by the pulse reflectometer, the end radii obtained from each of the trumpet leadpipe reconstructions were considered. The radii were rounded to the nearest $0.01 \mathrm{~mm}$. The results are shown in figure 12 which shows the occurrence of each radius. The graph for all the trumpet pipes had two distinct peaks either side of the average radius of $5.755 \mathrm{~mm}$. The curve for mandrel I peaks at $5.75 \mathrm{~mm}$ while mandrel II and III show peaks at $5.77 \mathrm{~mm}$ explaining the double peak observed in the curve for all the pipes. The spread of the results is $0.1 \mathrm{~mm}$, with all the results between 5.7 and $5.8 \mathrm{~mm}$. The curve for pipe 10a peaks at $5.73 \mathrm{~mm}$ and has a spread such that over $75 \%$ of the results for the final radius lie within $0.03 \mathrm{~mm}$; this is in line with the RMS results presented earlier and the assertion that differences between pipes as small as $0.03 \mathrm{~mm}$ can be detected.

Figure 12, however, only highlights differences; it does not address the question of how accurately the reflectometer measures the end radius of the pipe. The end radius of a 10a pipe was measured using calipers to be $5.75 \pm 0.025 \mathrm{~mm}$. The 32 reconstructions of pipe $10 \mathrm{a}$, shown in figure 12 , have a mean value of $5.742 \mathrm{~mm}$ for the end radius, with a standard deviation of $0.020 \mathrm{~mm}$, and none of the reconstructions has an end radius outside the range $5.7-5.8 \mathrm{~mm}$. Therefore, the evidence suggests that the uncertainty at the end of the trumpet leadpipe is likely to be no greater than $\pm 0.05 \mathrm{~mm}$. As expected, this is larger than the variation observed between reconstructions of the same pipe $(\sim 0.03 \mathrm{~mm})$ and the uncertainty with which the initial radius of the DC tube can be measured $( \pm 0.025 \mathrm{~mm})$. Despite being larger than the uncertainty which is obtained when calipers are used, this uncertainty is relatively small and suggests that the reflectometer is not only useful for observing small differences between objects, but it can also give an accurate measurement of the internal radius. It is expected that the value of $\pm 0.05 \mathrm{~mm}$ for the uncertainty in a measurement will increase with the length and size of the object being measured. It should also be noted that the reflectometer actually measures the cross-sectional area of an object and will therefore give an average value of the radius if the cross-section is not circular. Also, it is clearly not possible to obtain measurements with this accuracy in regions of the reconstruction where ripple is evident. 


\section{Discussion}

Generally the results have shown that the different leadpipes can be distinguished using the pulse reflectometer. However, in some reconstructions the ripple which can occur at any step within the pipe can obscure the actual radius of the pipe. In most cases the ripple is not large enough to cause a significant problem; however, three of the pipes produced a substantial amount of ripple relative to the radius differences which we are measuring. These are the three smallest pipes where the size of the step is largest: pipe 10, see figure 8; pipe R0, see figure 10, and pipe R2 (not presented). In figure 8 the ripple on the reconstruction of pipe 10 is evident for most of the reconstruction; it is only in the first $0.1 \mathrm{~m}$ that the level of ripple is unacceptable since we can no longer separate pipe 10 from pipes 12 and 14. The ripple on pipe R0 in figure 10 is worse than it is on pipe 10 and is unacceptable in that it renders almost the first half of the reconstruction unusable. With the exception of these three pipes, however, the ripple is not a real problem. Nevertheless, it would be useful if the problem of ripple could be improved. One possible approach is to use post-processing techniques to introduce fractional time delays so that the step between two interrogation cylinders coincides with the actual step. However, more work is required to asses the effectiveness of such an approach, both in terms of reducing the ripple and its computational efficiency.

\section{Conclusion}

A number of trumpet and cornet leadpipes have been studied using pulse reflectometry. By changing leadpipes a player can make musically significant changes to the playing properties of the instrument, but the differences between the pipes are small: typically the radius will differ by less than $0.1 \mathrm{~mm}$ between similar pipes. By comparing reconstructions of these leadpipes we have examined the ability of the reflectometer to distinguish between them. The reflectometer can typically detect differences in pipe radii greater than $0.03 \mathrm{~mm}$. Since all the pipes which are nominally the same size are not identical, the differences between replica pipes were also investigated. In most cases the differences were smaller than $0.03 \mathrm{~mm}$ and below the resolution of the reflectometer. In one case detectable differences were observed between two replicas of a cornet leadpipe. These differences could have appeared during the manufacture of the pipes, particularly in the bending of the cornet leadpipe, or they could be due to subsequent damage. The difference between the replica pipes was in general smaller than the differences observed between adjacent pipes which could easily be identified by the reflectometer. The accuracy of the measurements obtained from the reflectometer was also considered briefly. At the end of the pipe the uncertainty taken from a reconstruction was found to be $\pm 0.05 \mathrm{~mm}$. This is slightly less accurate than the measurement which could have been taken using calipers; however, the reflectometer has the advantage that it can make measurements in inaccessible places. Problems which still need to be addressed in reflectometry were also considered briefly; the main difficulty observed here is the ripple which appears in a reconstruction close to a radius step.
Overall, the reflectometer has been shown to be capable of detecting small differences between leadpipes which produce small but noticeable differences between the musical qualities of trumpets and cornets. Thus we conclude that the reflectometer is a useful tool for studying tubular musical instruments and also for diagnosing and pinpointing defects during instrument manufacture.

\section{Acknowledgment}

This work was supported by the Engineering and Physical Sciences Research Council, UK.

\section{References}

[1] Kausel W 2001 Bore reconstruction from measured acoustic input impedance; equipment, signal processing, algorithms and prerequisites ISMA2001: Proc. Int. Symp. on Musical Acoustics (Perugia, 2001) vol 2 pp 373-8

[2] Ware J A and Aki K 1969 Continuous and discrete inverse scattering problems in a stratified elastic medium: I. Planes at normal incidence J. Acoust. Soc. Am. 45 911-21

[3] Jackson A C, Butler J P, Millet E J, Hoppin F G and Dawson S V 1977 Airway geometry by analysis of acoustic pulse response measurements J. Appl. Physiol. 43 523-36

[4] Fredberg J J, Wohl M E B, Glass G M and Dorkin H L 1980 Airway area by acoustic reflections measured at the mouth J. Appl. Physiol. 48 749-58

[5] Brooks L J, Castile R G, Glass G M, Griscom N T, Wohl M E B and Fredberg J J 1984 Reproducibility and accuracy of airway area by acoustic reflection J. Appl. Physiol. 47 777-87

[6] Smith R A 1988 Its all in the bore! J. Int. Trumpet Guild 12 $42-5$

[7] Sharp D B and Campbell D M 1997 Leak detection in pipes using acoustic pulse reflectometry Acustica 83 560-6

[8] Campbell D M and MacGillivray T 1998 Reconstruction of the carnyx ISMA98: Proc. Int. Symp. on Musical Acoustics (Leavenworth, WA, 1998) pp 147-52

[9] Sharp D B, MacGillivray T J, Ring W, Buick J M and Campbell D M 1998 Acoustical comparison of bassoon crooks ISMA98: Proc. Int. Symp. on Musical Acoustics (Leavenworth, WA, 1998) pp 209-14

[10] Sharp D B and Buick J M 1999 Measurement of musical wind instruments using acoustic pulse reflectometry. Proc. Institute of Acoustics (London) vol 21 pp 191-8

[11] Sharp D B 1998 Increasing the length of tubular objects that can be measured using acoustic pulse reflectometry Meas. Sci. Technol. 9 1469-79

[12] MacGillivray T J and Greated C A 1999 Acoustic pulse reflectometry for the measurement of tubular structures Acustica 85 s 217

[13] Rosenhouse G, Amir N and Shimony U 1995 A discrete model for tubular acoustic systems with varying cross section-the direct and inverse problems. Part 1: theory Acustica $\mathbf{8 1}$ 450-62

[14] Rosenhouse G, Amir N and Shimony U 1995 A discrete model for tubular acoustic systems with varying cross section-the direct and inverse problems. Part 2: experiments Acustica 81 463-74

[15] Smith R A and Daniell G J 1976 Systematic approach to the correction of intonation in wind instruments Nature $\mathbf{2 6 2}$ 761-5

[16] http://www.smithwatkins.com

[17] Keefe D H 1984 Acoustical wave propagation in cylindrical ducts: transmission line parameter approximations for isothermal and nonisothermal boundary conditions J. Acoust. Soc. Am. 75 58-62 